\title{
Problemas de automación en las instalaciones de secado y molienda de las fábricas de cemento
}

\section{EXIGENCIAS FUNDAMENTALES A LAS INSTALACIONES DE SECADO Y MOLIENDA CONJUNTOS}

La fabricación de cemento tiene lugar en tres procesos parciales:

- la preparación de las primeras materias;

- el proceso de cocción;

- la preparación del cemento.

Estos tres procesos parciales están desacoplados dinámicamente de la corriente de materiales mediante almacenes de crudo y almacenes de clínker. Fluctuaciones en la producción de un proceso parcial no afectan a los siguientes procesos.

En el flujo de las corrientes de gases no existen estos almacenes desacoplados. Las perturbaciones en el paso de gases del horno rotatorio así como de los molinos de crudo y de cemento se transmiten, si no se prevén dispositivos de compensación, en la dirección de la corriente de gases, es decir, en sentido inverso al proceso parcial acoplado.

Por lo tanto, como dispositivos de compensación debieran de preverse siempre tuberías bypass o de desviación, a través de las cuales pudiera regularse constantemente la presión del gas entre dos procesos parciales. Una presión invariable antes o después de un proceso parcial, independientemente de las fluctuaciones de la corriente gaseosa dentro de un proceso parcial, simula el gran almacén desacoplado necesario que podría ser la atmósfera exterior.

Por existir otro almacén en la corriente de material, el almacén de grava detrás de la instalación trituradora, el proceso parcial de la preparación de las primeras materias se divide en dos secciones: la instalación de trituración B (incluyendo la homogeneización previa VH) y la instalación de secado-molienda MT (incluyendo la homogeneización de material crudo $\mathrm{RH}$ ) (fig. 1).

El problema de la instalación de secado-molienda únicamente puede plantearse relacionándolo con la instalación total. Las magnitudes de salida de la instalación de secadomolienda son las magnitudes de entrada del horno rotatorio. El proceso de cocción determina qué criterios de calidad, es decir, qué exigencias de calidad han de cumplir estas magnitudes al término de la instalación de secado-molienda. Las magnitudes decisi- 
vas en este sentido son la composición química, la finura-composición granulométrica y la humedad, que deben mantenerse constantes en el crudo.

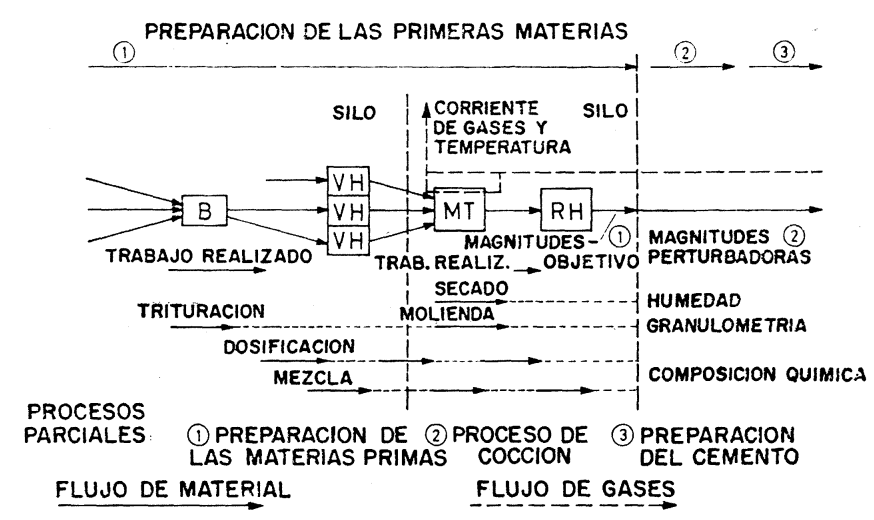
Fig. 1.-La instalación de secado-molienda MT dentro del pro-
ceso total.

La producción de la instalación total en un proceso en serie como la fabricación de cemento es igual a la capacidad del proceso parcial. La producción de la instalación de secado-molienda ha de corresponder, por lo tanto, como mínimo al rendimiento del proceso total, si es que en esta fase no ha de haber un estrechamiento del rendimiento. Pero si la instalación de molienda-secado es en alguna ocasión el "cuello de botella" del rendimiento -esto puede ocurrir en instalaciones productoras de cemento que, en general, se explotan muy por encima de su rendimiento nominal en el límite del elemento más débil-, entonces resulta que su producción máxima es una magnitud esencial en el planteamiento.

Además del cumplimiento de determinadas tolerancias en la calidad del crudo, en general se exige una rentabilidad a cada proceso parcial. En este sentido el consumo específico de energía eléctrica debe ser lo más reducido posible. Ahora bien, una instalación de secado-molienda, cuya capacidad es suficiente en servicio permanente, resultará demasiado pequeña si es que ha de aprovechar los tiempos de tarifas más favorables. La exigencia de rendimiento máximo resulta de nuevo ser una magnitud esencial en el planteamiento.

Para la instalación de secado-molienda, lo mismo que para el proceso total, vale la experiencia, a menudo confirmada, de que la rentabilidad alcanza un valor óptimo cuando la instalación funciona con máximo rendimiento más allá del período necesario y entonces se la para. Las reparaciones pueden efectuarse sin trabas. Este es otro motivo para aspirar al valor máximo en el rendimiento.

Las magnitudes de entrada del proceso de secado-molienda, tales como fluctuaciones en la composición química, finura (granulometría), humedad y dureza del material crudo a la salida de la prehomogeneización, son las magnitudes perturbadoras externas del propio proceso. Así como el proceso de cocción determina los márgenes de tolerancia máximos en el crudo, el proceso de secado-molienda determina estos márgenes para la materia prima. 
Así como existen estas magnitudes de objetivo y de perturbación en el flujo de material, también existen en la corriente de gases como datos de entrada al horno. Estos son: temperatura, presión, calor específico y flujo, de un lado, y mínima pérdida de calor y elevado punto de rocío, del otro.

A las magnitudes perturbadoras externas hay que añadir, en determinadas circunstancias, magnitudes perturbadoras internas, tales como: las alteraciones en el comportamiento de la instalación de molienda, las alteraciones en la resistencia de flujo, desgaste de los cuerpos molturadores y todas las perturbaciones del proceso en el curso normal.

El cumplir las magnitudes del objetivo prefijadas dentro de tolerancias razonables (magnitudes perturbadoras externas e internas), el comportamiento dinámico y estático de la propia instalación, el asegurar valores de medición adecuados y órganos de regulación idóneos y la estabilidad exigida, son determinantes para el montaje y el grado de inconveniente de una instalación de regulación automática.

Las magnitudes del objetivo hay que ponerlas a disposición de la instalación de regulación como magnitudes de medición. Con ayuda de magnitudes de ajuste o regulación idóneas se compensará la influencia de la magnitud perturbadora en las magnitudes consigna.

Estos problemas son muy distintos de una instalación a otra. El tratarlos ulteriormente hay que limitarlos, por lo tanto, a una determinada instalación de secado-molienda, en la que se realizaron los ensayos de regulación y mediciones necesarios.

\section{ANALISIS DE UNA INSTALACION ESPECIAL}

\subsection{Instalación}

Se analizó un "doble rotator" con una producción de 75 t/h, sobre el que se anticipan algunos datos para el montaje de la instalación.

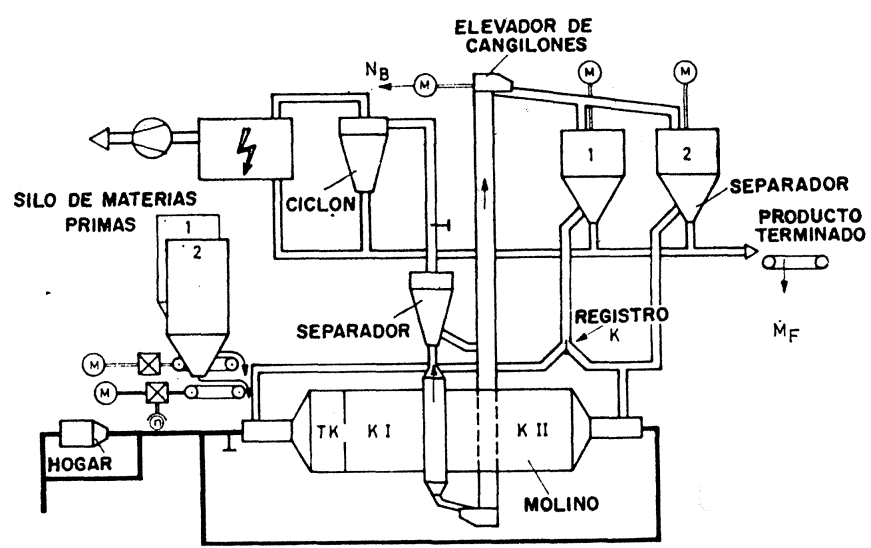

Fig. 2.-Esquema de la instalación analizada.

Dos cintas con un número de revoluciones regulado transportan las dos materias primas a mezclar desde los silos de grava a la entrada de la cámara de secado TK del molino tubular (fig. 2). El material se desplaza - por la cámara de secado TK, la cámara de molienda gruesa $\mathrm{K} \mathrm{I}$, el elevador (en parte paralelamente sobre el separador y elevador) y dos separadores centrífugos- a la báscula de cinta de salida para el producto terminado. La fracción de arenilla del separador derecho y una parte del izquierdo se vuelven a llevar a la cámara de molienda fina $\mathrm{K}$ II del molino tubular. 
Ya se dijo que en esta instalación era posible encontrar un ajuste fijo para el registro de distribución $\mathrm{K}$ que podía mantenerse en todas las gamas de rendimiento, de modo que ambas circulaciones del sistema doble pueden considerarse como un sistema sencillo. Es decir, que la interpretación que se dé a continuación vale también para molinos tubulares con retroceso sencillo del material.

La corriente de gases de secado se compone preferentemente del aire de salida del enfriador del horno que, mediante un hogar auxiliar, se pone a la temperatura necesaria. Los gases atraviesan las cámaras K I y K II desde las partes frontales; a continuación pasan por separador, ciclón, filtro eléctrico y exhaustor, que conduce la corriente de gases a la chimenea.

\subsection{Esquema de regulación}

El esquema regulador, definitivo, obtenido por mediciones y ensayos, se representa primeramente en un cuadro simplificado de la instalación. La argumentación de esta disposición se indicará luego (fig. 3).

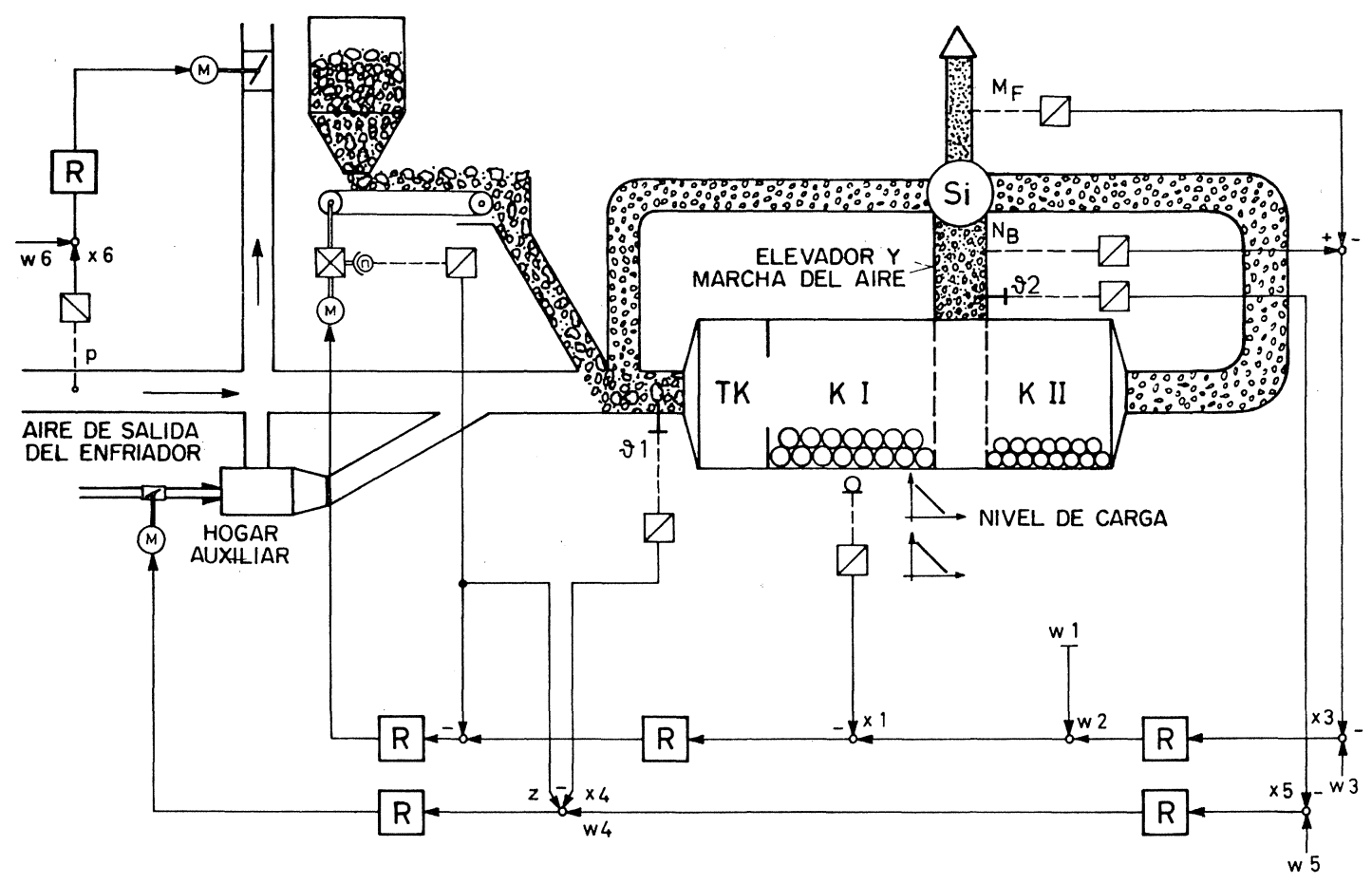

Fig. 3.-Esquema de regulación con circuitos reguladores indirectos para producción y humedad y un sistema regulador de presión para desacoplar el sistema del horno.

Las magnitudes que pretende la instalación son: producción máxima para todas las durezas de materias primas, composición uniforme de la mezcla cruda y humedad del crudo. La composición y la finura no se regulan, pero sí el rendimiento y la humedad con ayuda de procedimientos indirectos. 
El circuito regulador de producción está configurado en cascada triple: Dos circuitos reguladores de número de revoluciones sobre el plano inferior (representado en la figura 3 como un solo circuito regulador para la suma de ambas corrientes de material) ajustan el número de revoluciones de ambas cintas transportadoras, manteniéndolas en una relación constante, de modo que al ajustar la corriente total permanece constante la composición una vez mezclados. Es decir, la composición no se regula; hay que preocuparse únicamente de que los cambios de producción no actúen como magnitud perturbadora en la composición.

Los reguladores de números de revoluciones se dirigen desde un regulador de grado de llenado. El circuito regulador de grado de llenado averigua indirectamente el nivel de carga existente con ayuda de un oído eléctrico. Es decir, es un circuito regulador de intensidad de sonido o presión sonora.

El valor nominal del grado de llenado se indica previamente como suma del valor constante W 1 y de la señal de salida W 2 del regulador principal. Este regulador principal modifica la señal al fluctuar la de dureza de la primera materia, siempre a base de mantener corriente constante de retorno de las arenillas. La corriente-suma de las arenillas se mide, en este caso, indirectamente por la diferencia de la corriente del elevador (trabajo del elevador de cangilones) y la corriente del producto terminado.

¿Cómo funciona ahora esta cascada y qué requisitos ha de cumplir?

La cascada ha de ajustar, primeramente, la mayor producción posible en estado estacionario y, en segundo lugar, mostrar un buen comportamiento dinámico; ha de trabajar, por lo menos, de una forma estable.

Pasemos a la segunda exigencia (fig. 4). Con un ajuste en forma de saltos del número de revoluciones de la alimentación de material, o sea, de la magnitud de ajuste de la corriente de alimentación, reacciona primeramente el grado de llenado, a continuación la corriente del elevador y después la corriente del producto terminado. Es decir, la cascada está escalonada correctamente; la señal del grado de llenado trabaja buscando la estabilización.

Menciónese aquí que al variar la magnitud principal, la dureza de la materia prima, pueden dispararse las señales de respuesta en un orden de sucesión precisamente a la inversa del anteriormente señalado.

No obstante la regulación trabaja en cada caso de un modo estable, ya que la forma de intervención de una perturbación nunca puede influir decisivamente en la estabilidad de un circuito regulador. En lo que respecta a la magnitud de regulación materia primacorriente de alimentación, que es decisiva para la estabilidad, se escalona correctamente en cada caso.

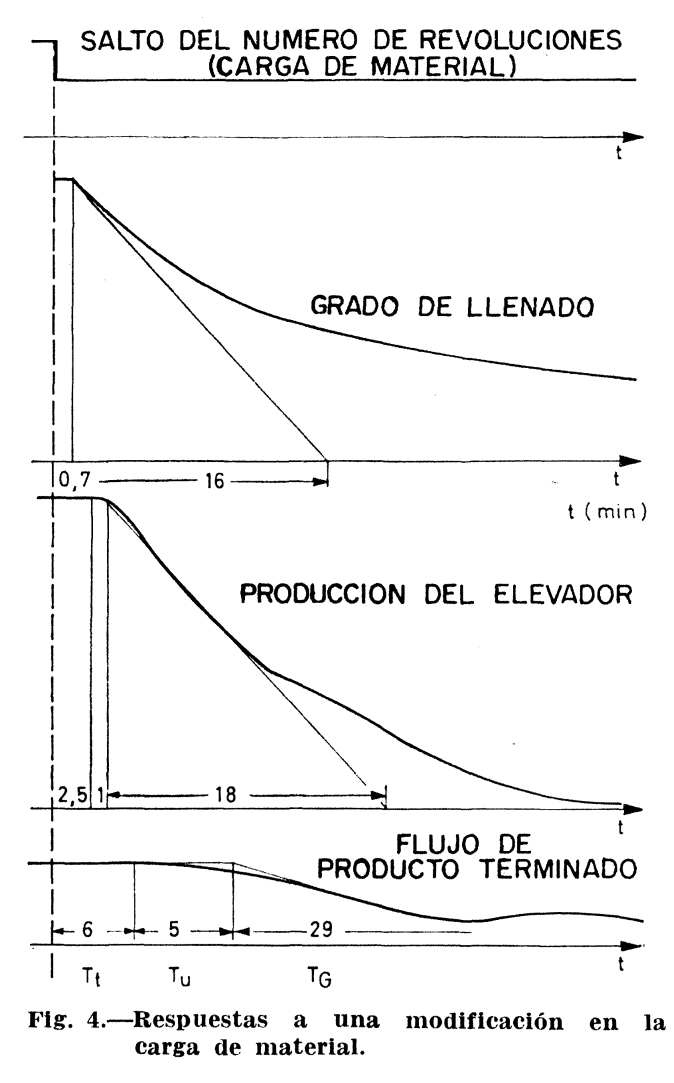


Para explicar el funcionamiento del circuito regulador debe discutirse, en primer lugar, el diagrama de la figura 5. Abajo, a la derecha, muestra el avance de la trituración, nueva superficie creada por unidad de tiempo en función del grado de llenado. En los puntos de partida del grado de llenado, es decir, con molino completamente lleno o completamente vacío, el avance de la trituración es cero. Entre estos puntos es siempre mayor que cero, con un máximo cuya altura y posición depende de la dureza de la primera materia. Al pasar de material duro a material más blando aumenta el avance de trituración, desplazándose el óptimo, probablemente, hacia mayores grados de llenado.

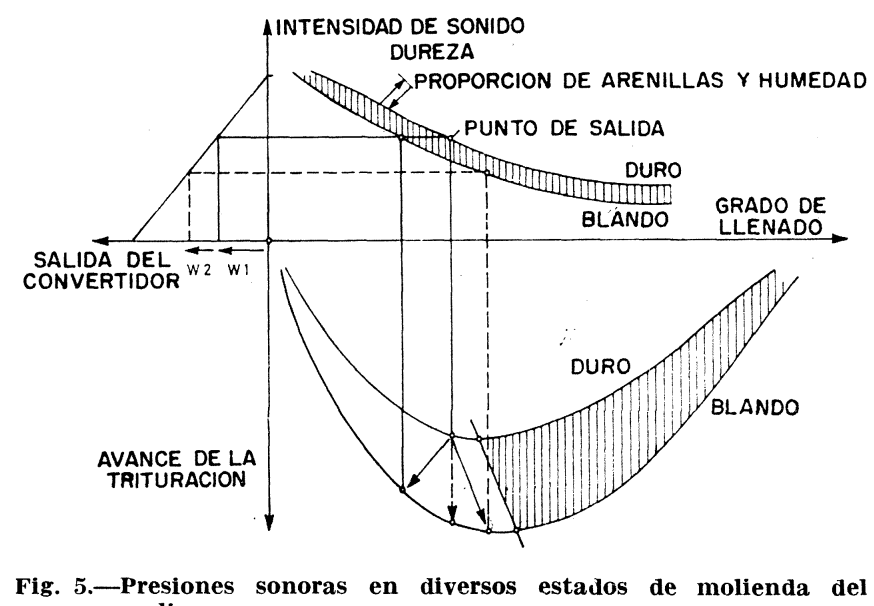
molino.

El diagrama muestra además la presión sonora en función del grado de llenado y de la naturaleza del material. La presión sonora disminuye al aumentar el grado de llenado y existir mayor proporción de arenillas; aumenta al crecer la dureza de la materia prima. En el diagrama presente se supuso que la dureza no tuvo en cuenta la influencia de la mayor proporción de arenillas.

El diagrama muestra, finalmente, la dependencia de la corriente de salida del convertidor electroacústico de la presión sonora. La curva característica negativa compensa la dependencia negativa de la presión sonora del grado de llenado del molino.

Los grados de llenado óptimos para materias primas extremadamente duras y blandas se conocen por ensayos previos en la zona de intensidad de sonido. Estos valores no permiten una indicación exacta del grado de llenado efectivo, ya que no se pueden abarcar cuantitativamente los falseamientos producidos por la influencia de la dureza y las arenillas. Pero para la regulación este hecho no tiene, sin embargo, excesiva importancia, pues el. regulador de grado de llenado sólo elabora la señal de llenado en la zona de la presión sonora y el óptimo ya se conoce en dicha zona.

Es prácticamente imposible marchar en la zona de la derecha del óptimo, o sea, hacia mayores grados de llenado. Como consecuencia de la inversión de los signos de la curva caracteristica, un regulador normal reaccionaría erróneamente al sobrepasarse el óptimo. Por lo tanto se determina con W 1 el mayor grado de llenado posible para material duro, teniendo en cuenta una distancia de seguridad que corresponde al rendimiento efectivo. Una regulación constante del grado de llenado, con material más blando alejaría mucho 
el punto de rendimiento de la instalación del punto posible. Unicamente aumentando el valor nominal del grado de llenado en la cantidad W 2 puede hacer funcionar la instalación cerca del nuevo punto efectivo.

La magnitud W 2 la suministra el regulador principal que, al pasar a un material más blando, observa una disminución de la corriente de retorno de las arenillas y aumenta su señal de salida y con ella el grado de llenado hasta que la corriente de retorno de las arenillas vuelve al valor primitivo.

El regulador principal lleva un tope superior, o sea, que la magnitud W 2 es limitada. De este modo se le quita al regulador principal la posibilidad de aumentar demasiado el grado de llenado y llegar a saturar el molino.

La regulación constante de la corriente de retorno de las arenillas realiza, por lo tanto, una regulación indirecta del rendimiento máximo posible. La magnitud de ajuste del regulador principal es el grado de llenado, que se ajusta siempre de manera que se alcance la máxima producción posible.

Obsérvese que la citada regulación de la producción influye en la finura del material crudo en la dirección deseada. Con materiales duros se establece un grado de llenado menor, y así se realiza más trabajo de molturación con períodos de tratamiento más prolongados.

El circuito regulador de temperatura (fig. 3) fue previsto para que, indirectamente, se mantuviera una humedad constante en el crudo. El hogar auxiliar sirve de elemento corrector, que se ajusta inmediatamente a través de un servorregulador cuando varía el flujo de material (magnitud perturbadora Z) o cuando cambia la temperatura del aire del enfriador (magnitud de regulación auxiliar X 4).

El regulador piloto interviene a través de su salida W 4, cuando, como consecuencia de una variación en la humedad del material, la temperatura de los gases X 5 después del molino discrepa de su valor teórico W 5 , medida en el separador.

Como condición marginal para el montaje de una regulación indirecta de la humedad con arreglo a este método, sirve el que sea constante la corriente de gases por el molino, puesto que de esta corriente y de su temperatura depende el efecto de secado en el molino.

La corriente de gases se mantiene constante en el molino tubular cuando la depresión es constante en el sistema de molienda, pues la resistencia del flujo del sistema es prácticamente independiente del grado de llenado del molino. Un regulador de presión mantiene constante, por lo tanto, la presión a la entrada del molino con ayuda de un órgano de mando en una tubería bypass.

Este circuito regulador de presión evita - como ya se indicó antes- no sólo las fluctuaciones de presión o bien fluctuaciones de corriente de gases del sistema del horno en el molino, sino que protege también el sistema del horno, de fluctuaciones en la presión $\mathrm{y}$ en el flujo de gases del molino.

\subsection{Problemas de medida}

El principal problema en la regulación de todos los procesos parciales de la fabricación del cemento es la metrología, el cual es también el caso en la instalación reguladora de molienda y secado. 
Para la regulación de la humedad no se da ningún valor de medición directa de la humedad del crudo. Se está a punto de elegir la temperatura del polvo molido como magnitud de medida indirecta, pero ésta no es fácil de medir. La temperatura de los gases de salida es relativamente fácil de medir, pero el valor medido puede falsearse y demorarse debido a formación de colgaduras o pegaduras.

Para la medida de la producción no se da una señal de medida directa para el flujo de retorno de las arenillas, si no da la señal una báscula de cinta, la cual es cara y difícil de montar ulteriormente. La medida indirecta de la carga del elevador, descontando el flujo de material terminado, no es muy exacta, ya que el trabajo del elevador da un valor de base grande en la marcha en vacío y una señal útil relativamente reducida. Además, deben compensarse las fluctuaciones superpuestas del paso de los cangilones, y la señal ha de demorarse para que la señal útil esté en fase con la señal del producto terminado.

Se ve que son necesarias muchas experiencias, por parte del ingeniero de ensayos, para tener en cuenta todas estas relaciones.

Del mismo modo, la medida del grado de llenado con ayuda del oído eléctrico representa otra medición indirecta, que tiene un largo desarrollo tras de sí. Las figuras 6 y 7 reproducen los resultados de algunas medidas. En la figura 6 se indica la presión sonora en función del lugar de emplazamiento longitudinal del micrófono en el molino con ángulo constante (lado de caída de las bolas) y distancia constante $(40 \mathrm{~mm})$ de la envolvente del molino. Las curvas para un sonido constante de 30,40 y $50 \%$ del valor teórico $\mathrm{W} 1$ del regulador, alcanzan su máximo a $3 \mathrm{~m}$ de distancia de la entrada del molino.

Con $1.000 \mathrm{~Hz}$ se alcanza la máxima intensidad (fig. 7). La curva para $1.000 \mathrm{~Hz}$ transcurre, en función del lugar de emplazamiento longitudinal, prácticamente paralela a las curvas de sonido global (fig. 6).

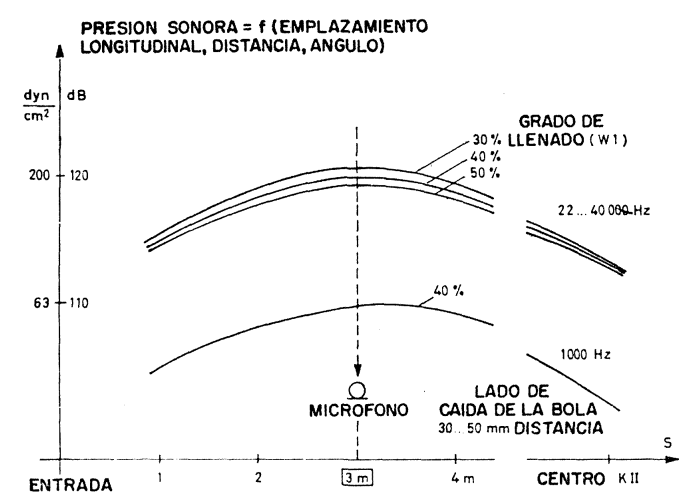

Fig. 6.-Marcha de la presión sonora a lo largo del molino.

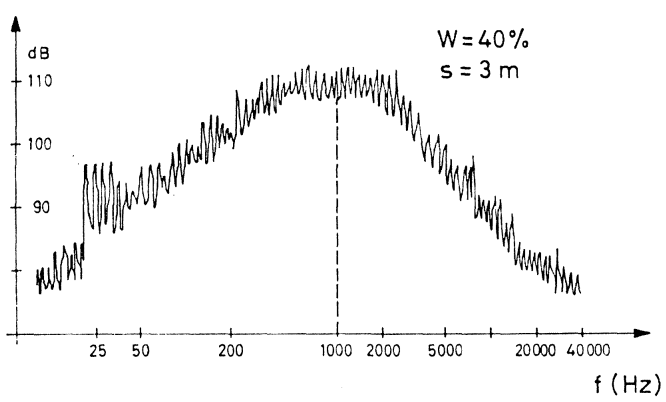

Fig. 7.-Análisis de la frecuencia del ruido de los molinos.

Como señal reguladora se utiliza una medida del sonido global. El micrófono se halla al máximo de la intensidad de sonido a $3 \mathrm{~m}$ de la entrada y $40 \mathrm{~mm}$ de distancia sobre el lado de caída de las bolas.

Las anteriores indicaciones sobre el tema metrología parece que tienen por finalidad enumerar las dificultades por las cuales los valores medidos pueden parecer inseguros. Pero 
en realidad subrayan la necesidad de disponer de experiencias y sacan a colación el tener que realizar observaciones y análisis profundos, ya que sin una metrología confiable no pueden existir resultados de regulación satisfactorios.

\section{ESQUEMA GENERAL DE REGULACION DE LA INSTALACION DE SECADO-MOLIENDA}

A todas las instalaciones de secado-molienda, independientemente del tipo de construcción, se les exige prácticamente las mismas condiciones. Siempre se trata de orientar las propiedades físicas y químicas de las corrientes de material y gases producidas hacia los valores prefijados. Así, es de esperar que instalaciones diferentes acusen características iguales, incluso aunque las características constructivas difieran mucho de unas a otras, ya que siempre se vuelve a la estructura fundamental del método. Esta estructura de las instalaciones, juntamente con el comportamiento dinámico de los diversos canales de acción, son decisivos para contestar a todas las preguntas de la técnica de la regulación.

Las comprobaciones hechas en el capítulo anterior en una instalación especial pueden, en su mayor parte, transmitirse a otras instalaciones. En la instalación de molienda-secado de la figura 8 existen, como en el horno rotatorio, dos corrientes continuas -la corriente de material y la corriente de gases- portadoras del proceso. Estas corrientes vuelven en parte al proceso. Por el lado del material todas las instalaciones tienen una corriente de retorno de arenillas del separador. En instalaciones con molinos verticales esta corriente no es accesible de acuerdo con la técnica de la regulación.

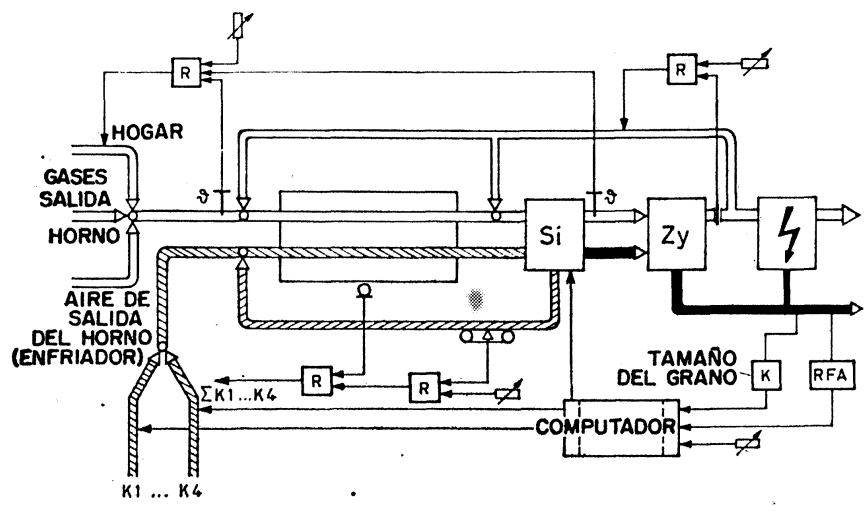

Fig. 8.-Esquema general para instalaciones de molienda-secado con una instalación de regulación especial.

Las corrientes de retorno de los gases se desvían, generalmente, después del ciclón y se llevan nuevamente a la entrada del molino (molino vertical) o delante y detrás del molino (instalación de molienda con molino ventilado). En vez de las corrientes de retorno se establecen corrientes bypass siempre hacia adelante.

En todos los casos es válido, lo mismo que en el horno rotatorio, mantener constante la corriente de gases a través del molino. Una corriente de los gases del molino constante produce, por ejemplo, un transporte y una alimentación del separador uniforme, consti- 
tuyendo la condición previa fundamental para establecer regulaciones indirectas, tales como: la de la humedad a través de la temperatura de los gases o la del grado de llenado del molino vertical a través de la presión diferencial.

El flujo de material al molino hay que acomodarlo, normalmente, a la posible capacidad de molienda del molino. Esta capacidad de molienda, expresada en peso por unidad de tiempo, varía con la dureza y la humedad de la primera materia. Los puntos de medida decisivos, que permiten una afirmación sobre si el proceso de molienda transcurre cerca del límite de producción, se hallan siempre dentro del depósito circulante molino-descarga-separador-transporte de retorno de arenillas-molino; tales son: la medida del grado de llenado en el molino, la medida de rendimiento en el elevador de cangilones o la corriente de retorno de las arenillas.

Además del ajuste de las dos corrientes del proceso, la instalación de regulación posee también parámetros para mando de estas corrientes: finura (granulometría), humedad, composición química y temperatura de los gases de salida. Es decir, el sistema es siempre un sistema de regulación múltiple, en el que varias magnitudes de salida (magnitudes de regulación) hay que regularlas con ayuda de, por lo menos, otras tantas magnitudes de entrada (magnitudes de mando), por lo que, para cada magnitud de salida, hay que determinar la correspondiente magnitud de entrada. En esta coordinación se tendrán en cuenta los siguientes puntos de vista:

Cada magnitud de regulación debe ser modificada por la magnitud de mando asignada a ella, con una amplificación lo más elevada posible y un tiempo de demora reducido. El comportamiento en la transmisión ha de ser lo más lineal posible. La influencia de esta magnitud de mando en otras magnitudes de regulación debe de ser pequeña y los acoplamientos entre los diversos circuitos de regulación deben ser mínimos.

El número de magnitudes de regulación se determina con el número de magnitudes establecidas con objetivo, a no ser que varias de estas magnitudes sean ya suficientemente constantes sin intervención de la regulación.

En las magnitudes a regular se analizará, en cada caso, la posibilidad para incluir la metrología directa o indirecta.

- Cada magnitud de regulación establecida ha de poderse abarcar con una técnica de medida exacta, rápida y con seguridad de servicio. Si en su lugar se establece una magnitud semejante a ella (método indirecto), la relación será lo más inequívoca posible e invariable con el tiempo.

En relación con esto, también hay que conceder la necesaria atención a las magnitudes de mando:

- Todos los elementos de mando serán de funcionamiento seguro.

- Su comportamiento de intervención será lo más lineal posible.

La selección de las magnitudes de regulación y mando es un proceso iterativo, en el que hay que considerar constantemente magnitudes objetivo, problemas de medida, problemas de mando, estructura y comportamiento de la instalación y las magnitudes perturbadoras; por ello, es necesario aprovechar al máximo las posibilidades de las regulaciones adicionales de las magnitudes perturbadoras. Para ello se requiere un sistema de regulación flexible, en el que se puedan realizar discrecionalmente combinaciones de señales. Un sistema que sólo permite formar circuitos de regulación individuales, no es suficiente. 
El esquema de una instalación de secado-molienda expuesto en la figura 8 contiene una propuesta especial para una instalación reguladora y, por ejemplo, puede montarse en una instalación de molienda con arrastre por aire. Para una mejor visibilidad, sólo se han establecido los órganos de medida y los órganos de mando.

Existen dos circuitos de regulación convencionales que determinan la corriente de gases por el molino y el flujo de material. Otro circuito regulador convencional de la temperatura obliga a mantenerse uniforme la humedad del material crudo. La regulación de finura constante con ayuda del número de revoluciones del separador y la regulación de la composición del crudo (medida con una instalación de fluorescencia de rayos X) por regulación del flujo de entrada en el molino, se resuelven con ayuda de un computador digital.

El montaje de esta instalación de regulación, con la inclusión de los RFA, de la medida del tamaño de grano y del computador, inicia un circuito de nuevos problemas funcionales y dinámicos, pero que no se han de analizar en el ámbito de este trabajo.

Un computador es necesario para regular la composición del crudo, pero además puede centralizar las demás misiones de regulación dibujadas en la figura 8. Como quiera que con un fallo en el computador fallaría toda la instalación reguladora, son evidentes las ventajas de la disposición descentralizada dibujada en la citada figura. Los demás aparatos continúan trabajando al fallar un aparato de la instalación.

El circuito regulador de la mezcla se halla ampliamente desacoplado de los demás circuitos reguladores. Un fallo de este circuito prácticamente no perturba el desarrollo de la regulación de los otros circuitos. Esta independencia relativamente grande del circuito regulador de la mezcla justifica el no tratar más minuciosamente en este trabajo el circuito de problemas instalación RFA y computador.

Como final de estas explicaciones vamos a establecer dos ideas generalmente válidas, que son decisivas precisamente en la automación de instalaciones complejas, pero que, sin embargo, no se tienen lo suficientemente en cuenta por muchos fabricantes y usuarios.

Por un lado, se encuentra la idea de que no toda instalación es capaz de ser regulada. Frecuentemente el recorrido de regulación, es decir, la instalación, tiene un comportamiento tan malo que no es posible conseguir un resultado de regulación satisfactorio. Por tanto, los constructores de instalaciones tienen que intentar buenas propiedades de regulación al construir la instalación.

Para la segunda determinación es válida la experiencia de que una instalación de regulación, por bien proyectada y montada que esté, cuando se la conecta, en general no funciona inmediatamente. Una instalación de regulación múltiple, en la que además existen dificultades de medida, siempre debe ponerse en marcha por un ingeniero experimentado, o sea que esté en condiciones de solucionar todos los problemas que se le presenten: desde el proceso, a través de la técnica de regulación, hasta la electrónica de los aparatos.

\section{R E S U M E N}

En el diseño de los sistemas de control de las plantas de molienda es necesario, en primer lugar, establecer los objetivos a alcanzar, en forma de magnitudes. Junto a las exigen- 
cias en cuanto a las características de calidad del crudo se refiere, existen las referentes al logro de la máxima producción del molino.

El autor describe el conjunto de dispositivos de regulación instalados en cascada triple sobre una instalación de secado-molienda con doble-rotator. Esta regulación rige la producción máxima de una manera indirecta, a través de la regulación del retorno de los gruesos del separador.

La molturabilidad variable de la materia prima representa una magnitud perturbadora. Esto se compensa con ayuda de la medida del grado de llenado con oído eléctrico. El regulador principal modifica entonces el valor patrón del nivel sonoro, en función del reciclado de grúesos, indicando esto último la variación en la molturabilidad.

A pesar de las diferencias de diseño, las diferentes instalaciones de molienda se basan en esquemas de proceso similares. Los problemas similares que se presentan y la experiencia lograda en todos ellos son aplicables a las diferentes plantas. 